\title{
Revisiting Lepton Flavor Universality in $B$ Decays
}

\author{
Ferruccio Feruglio, ${ }^{1,2}$ Paride Paradisi, ${ }^{1,2}$ and Andrea Pattori ${ }^{1,3}$ \\ ${ }^{1}$ Dipartimento di Fisica e Astronomia "G. Galilei”, Università di Padova, I-35100 Padova, Italy \\ ${ }^{2}$ Istituto Nazionale Fisica Nucleare, Sezione di Padova, I-35131 Padova, Italy \\ ${ }^{3}$ Physik-Institut, Universität Zürich, CH-8057 Zürich, Switzerland
}

(Received 22 June 2016; published 3 January 2017)

\begin{abstract}
Lepton flavor universality (LFU) in $B$ decays is revisited by considering a class of semileptonic operators defined at a scale $\Lambda$ above the electroweak scale $v$. The importance of quantum effects, so far neglected in the literature, is emphasized. We construct the low-energy effective Lagrangian taking into account the running effects from $\Lambda$ down to $v$ through the one-loop renormalization group equations (RGEs) in the limit of exact electroweak symmetry and QED RGEs from $v$ down to the $1 \mathrm{GeV}$ scale. The most important quantum effects turn out to be the modification of the leptonic couplings of the vector boson $Z$ and the generation of a purely leptonic effective Lagrangian. Large LFU breaking effects in $Z$ and $\tau$ decays and visible lepton flavor violating effects in the processes $\tau \rightarrow \mu \ell \ell, \tau \rightarrow \mu \rho, \tau \rightarrow \mu \pi$, and $\tau \rightarrow \mu \eta^{(\prime)}$ are induced.
\end{abstract}

DOI: 10.1103/PhysRevLett.118.011801

I. Introduction.-Lepton flavor universality (LFU) tests are among the most powerful probes of the standard model (SM) and, in turn, of new physics (NP) effects. In recent years, experimental data in $B$ physics hinted at deviations from the SM expectations, both in charged-current as well as neutral-current transitions. The statistically most significant data are (i) an overall $3.9 \sigma$ violation from the $\tau / \ell$ universality $(\ell=\mu, e)$ in the charged-current $b \rightarrow c$ decays [1-4]

$$
\begin{aligned}
R_{D^{(*)}}^{\tau / \ell} & =\frac{\mathcal{B}\left(\bar{B} \rightarrow D^{(*)} \tau \bar{\nu}\right)_{\exp } / \mathcal{B}\left(\bar{B} \rightarrow D^{(*)} \tau \bar{\nu}\right)_{\mathrm{SM}}}{\mathcal{B}\left(\bar{B} \rightarrow D^{(*)} \ell \bar{\nu}\right)_{\exp } / \mathcal{B}\left(\bar{B} \rightarrow D^{(*)} \ell \bar{\nu}\right)_{\mathrm{SM}}}, \\
R_{D}^{\tau / \ell} & =1.37 \pm 0.17, \quad R_{D^{*}}^{\tau / \ell}=1.28 \pm 0.08,
\end{aligned}
$$

and (ii) a $2.6 \sigma$ deviation from $\mu / e$ universality in the neutralcurrent $b \rightarrow s$ transition [5]

$$
R_{K}^{\mu / e}=\frac{\mathcal{B}\left(B \rightarrow K \mu^{+} \mu^{-}\right)_{\exp }}{\mathcal{B}\left(B \rightarrow K e^{+} e^{-}\right)_{\exp }}=0.745_{-0.074}^{+0.090} \pm 0.036,
$$

while $\left(R_{K}^{\mu / e}\right)_{\mathrm{SM}}=1$ up to few $\%$ corrections [6].

As argued in Refs. [7-10], by means of global-fit analyses, the explanation of the $R_{K}^{\mu / e}$ anomaly favors an effective four-fermion operator involving left-handed currents, $\left(\bar{s}_{L} \gamma_{\mu} b_{L}\right)\left(\bar{\mu}_{L} \gamma_{\mu} \mu_{L}\right)$. This naturally suggests to account also for the charged-current anomaly through a left-handed operator $\left(\bar{c}_{L} \gamma_{\mu} b_{L}\right)\left(\bar{\tau}_{L} \gamma_{\mu} \nu_{L}\right)$, which is related to $\left(\bar{s}_{L} \gamma_{\mu} b_{L}\right)\left(\bar{\mu}_{L} \gamma_{\mu} \mu_{L}\right)$ by the $S U(2)_{L}$ gauge symmetry [11]. Clearly, this picture might work only provided NP couples much more strongly to the third generation than to the first two. Such a requirement can be naturally accomplished in two ways: (i) assuming that NP is coupled in the interaction basis only to the third generation of quarks and leptonscouplings to lighter generations are then generated by the misalignment between the mass and the interaction bases through small flavor mixing angles [12] - and (ii) if NP couples to different fermion generations proportionally to their mass squared [13]. In scenario (i), LFU violation necessarily implies lepton flavor violating (LFV) phenomena. The same is not true in scenario (ii) if the lepton family numbers are preserved.

In this Letter, we revisit the LFU in $B$ decays focusing on a class of semileptonic operators defined above the electroweak scale $v$ and invariant under the full SM gauge group along the lines of Refs. [11-17]. The main new development of our study is the construction of the low-energy effective Lagrangian taking into account the running of the Wilson coefficients of a suitable operator basis and the matching conditions when mass thresholds are crossed. The running effects from the NP scale $\Lambda$ down to the electroweak scale are included through the one-loop renormalization group equations (RGEs) in the limit of exact electroweak symmetry [18]. From the electroweak scale down to the $1 \mathrm{GeV}$ scale, we use the QED RGEs. By explicit calculations, we have checked that the scale dependence of the RGE contributions from gauge and top Yukawa interactions cancels with that of the matrix elements in the relevant physical amplitudes. Such a program has not been carried out in the literature so far, and it has significant implications on the conclusions of Refs. [1117]. The most important quantum effects turn out to be the modification of the leptonic couplings of the vector boson $Z$ and the generation of a purely leptonic effective Lagrangian. As a result, large LFV and LFU breaking effects in $Z$ and $\tau$ decays are induced.

II. Effective Lagrangians. - If the NP contributions originate at a scale $\Lambda \gg v$, in the energy window above $v$ and below $\Lambda$, the NP effects can be described by an effective Lagrangian $\mathcal{L}=\mathcal{L}_{\mathrm{SM}}+\mathcal{L}_{\mathrm{NP}}$ invariant under the $\mathrm{SM}$ gauge group. Here we assume that NP is dominated by

$$
\begin{aligned}
\mathcal{L}_{\mathrm{NP}}= & \frac{C_{1}}{\Lambda^{2}}\left(\bar{q}_{3 L} \gamma^{\mu} q_{3 L}\right)\left(\bar{e}_{3 L} \gamma_{\mu} \ell_{3 L}\right) \\
& +\frac{C_{3}}{\Lambda^{2}}\left(\bar{q}_{3 L} \gamma^{\mu} \tau^{a} q_{3 L}\right)\left(\bar{\ell}_{3 L} \gamma_{\mu} \tau^{a} \ell_{3 L}\right) .
\end{aligned}
$$


We move from the interaction to the mass basis through the unitary transformations

$$
\begin{gathered}
u_{L} \rightarrow V_{u} u_{L}, \quad d_{L} \rightarrow V_{d} d_{L}, \quad V_{u}^{\dagger} V_{d}=V, \\
\nu_{L} \rightarrow U_{e} \nu_{L}, \quad e_{L} \rightarrow U_{e} e_{L},
\end{gathered}
$$

where $V$ is the CKM matrix, and neutrino masses have been neglected. We get

$$
\begin{aligned}
\mathcal{L}_{\mathrm{NP}}= & \frac{1}{\Lambda^{2}}\left[\left(C_{1}+C_{3}\right) \lambda_{i j}^{u} \lambda_{k l}^{e}\left(\bar{u}_{L i} \gamma^{\mu} u_{L j}\right)\left(\bar{\nu}_{L k} \gamma_{\mu} \nu_{L l}\right)\right. \\
& +\left(C_{1}-C_{3}\right) \lambda_{i j}^{u} \lambda_{k l}^{e}\left(\bar{u}_{L i} \gamma^{\mu} u_{L j}\right)\left(\bar{e}_{L k} \gamma_{\mu} e_{L l}\right) \\
& +\left(C_{1}-C_{3}\right) \lambda_{i j}^{d} \lambda_{k l}^{e}\left(\bar{d}_{L i} \gamma^{\mu} d_{L j}\right)\left(\bar{\nu}_{L k} \gamma_{\mu} \nu_{L l}\right) \\
& +\left(C_{1}+C_{3}\right) \lambda_{i j}^{d} \lambda_{k l}^{e}\left(\bar{d}_{L i} \gamma^{\mu} d_{L j}\right)\left(\bar{e}_{L k} \gamma_{\mu} e_{L l}\right) \\
& \left.+2 C_{3}\left(\lambda_{i j}^{u d} \lambda_{k l}^{e}\left(\bar{u}_{L i} \gamma^{\mu} d_{L j}\right)\left(\bar{e}_{L k} \gamma_{\mu} \nu_{L l}\right)+\text { H.c. }\right)\right],
\end{aligned}
$$

where

$\lambda_{i j}^{q}=V_{q 3 i}^{*} V_{q 3 j}, \quad \lambda_{i j}^{e}=U_{e 3 i}^{*} U_{e 3 j}, \quad \lambda_{i j}^{u d}=V_{u 3 i}^{*} V_{d 3 j}$,

with $q=u, d$. These matrices are redundant since they satisfy the relations $\lambda^{u}=V \lambda^{d} V^{\dagger}$ and $\lambda^{u d}=V \lambda^{d}$. We also observe that $\lambda^{f}$ are Hermitian rank-1 matrices satisfying $\lambda^{f} \lambda^{f}=\lambda^{f}$ and $\operatorname{tr} \lambda^{f}=1$. In summary, the free parameters of our Lagrangian are the ratios $\left(C_{1,3}\right) / \Lambda^{2}$ and the two matrices $\lambda^{d}$ and $\lambda^{e}$.

Starting from the effective Lagrangian $\mathcal{L}_{\mathrm{NP}}$ at the scale $\Lambda$, at lower energies an effective Lagrangian is induced by RGE and by integrating out the heavy degrees of freedom. We will detail this procedure elsewhere. Here we summarize our results obtained in a leading logarithmic approximation.

The effective Lagrangian describing the semileptonic processes $b \rightarrow s \ell \ell$ and $b \rightarrow s \nu \nu$ is [19]

$\mathcal{L}_{\text {eff }}^{\mathrm{NC}}=\frac{4 G_{F}}{\sqrt{2}} \lambda_{b s}\left(C_{\nu}^{i j} \mathcal{O}_{\nu}^{i j}+C_{9}^{i j} \mathcal{O}_{9}^{i j}+C_{10}^{i j} \mathcal{O}_{10}^{i j}\right)+$ H.c.,

where $\lambda_{b s}=V_{t b} V_{t s}^{*}$ and the operators $\mathcal{O}_{\nu}$ and $\mathcal{O}_{9,10}$ read

$$
\begin{gathered}
\mathcal{O}_{\nu}^{i j}=\frac{e^{2}}{(4 \pi)^{2}}\left(\bar{s}_{L} \gamma_{\mu} b_{L}\right)\left(\bar{\nu}_{i} \gamma^{\mu}\left(1-\gamma_{5}\right) \nu_{j}\right), \\
\mathcal{O}_{9}^{i j}=\frac{e^{2}}{(4 \pi)^{2}}\left(\bar{s}_{L} \gamma_{\mu} b_{L}\right)\left(\bar{e}_{i} \gamma^{\mu} e_{j}\right), \\
\mathcal{O}_{10}^{i j}=\frac{e^{2}}{(4 \pi)^{2}}\left(\bar{s}_{L} \gamma_{\mu} b_{L}\right)\left(\bar{e}_{i} \gamma^{\mu} \gamma_{5} e_{j}\right) .
\end{gathered}
$$

By matching $\mathcal{L}_{\text {eff }}^{\mathrm{NC}}$ with $\mathcal{L}_{\mathrm{NP}}$, we obtain

$$
\begin{gathered}
C_{9}^{i j}=-C_{10}^{i j}=\frac{4 \pi^{2}}{e^{2} \lambda_{b s}} \frac{v^{2}}{\Lambda^{2}}\left(C_{1}+C_{3}\right) \lambda_{23}^{d} \lambda_{i j}^{e}+\ldots, \\
C_{\nu}^{i j}=\frac{4 \pi^{2}}{e^{2} \lambda_{b s}} \frac{v^{2}}{\Lambda^{2}}\left(C_{1}-C_{3}\right) \lambda_{23}^{d} \lambda_{i j}^{e}+\ldots,
\end{gathered}
$$

where the dots stand for RGE-induced terms which are always subdominant unless $C_{1}=-C_{3}$ or $C_{1}=C_{3}$. The latter condition, which can be realized in scenarios with vector leptoquark mediators [17], received a lot of attention in the literature as it allows us to avoid the $B \rightarrow K^{(*)} \nu \bar{\nu}$ constraint. We point out that such condition is not stable under quantum corrections. RGE effects driven by the gauge interactions generate a rather large correction to $c_{-}=C_{1}-C_{3}$ at the electroweak scale

$$
\delta c_{-} \approx-0.03 C_{3} \log \left(\frac{\Lambda}{m_{Z}}\right),
$$

which is of order $\left|\delta c_{-}\right| \sim 0.1$ for $C_{3}=1$ and $\Lambda \sim \mathrm{TeV}$.

The effective Lagrangian relevant for charged-current processes like $b \rightarrow c \ell \nu$ is given by

$$
\mathcal{L}_{\text {eff }}^{\mathrm{CC}}=-\frac{4 G_{F}}{\sqrt{2}} V_{c b}\left(C_{L}^{c b}\right)_{i j}\left(\bar{c}_{L} \gamma_{\mu} b_{L}\right)\left(\bar{e}_{L i} \gamma^{\mu} \nu_{L j}\right)+\text { H.c. }
$$

where the coefficient $\left(C_{L}^{c b}\right)_{i j}$ reads

$$
\left(C_{L}^{c b}\right)_{i j}=\delta_{i j}-\frac{v^{2}}{\Lambda^{2}} \frac{\lambda_{23}^{u d}}{V_{c b}} C_{3} \lambda_{i j}^{e} .
$$

One of the effects due to $\mathcal{L}_{\mathrm{NP}}$ is the modification of the leptonic couplings of the vector bosons $W$ and $Z$. Focusing on the $Z$ couplings, which are the most tightly constrained by the experimental data, we find that

$\mathcal{L}_{Z}=\frac{g_{2}}{c_{W}} \bar{e}_{i}\left(Z g_{\ell L}^{i j} P_{L}+Z g_{\ell R}^{i j} P_{R}\right) e_{j}+\frac{g_{2}}{c_{W}} \bar{\nu}_{L i} Z g_{\nu L}^{i j} \nu_{L j}$,

where $g_{f L, R}=g_{f L, R}^{\mathrm{SM}}+\Delta g_{f L, R}, c_{W}=\cos \theta_{W}$, and

$\Delta g_{\ell L}^{i j} \simeq \frac{v^{2}}{\Lambda^{2}}\left(3 y_{t}^{2} c_{-} \lambda_{33}^{u} L_{t}+g_{2}^{2} C_{3} L_{z}+\frac{g_{1}^{2}}{3} C_{1} L_{z}\right) \frac{\lambda_{i j}^{e}}{16 \pi^{2}}$,

$\Delta g_{\nu L}^{i j} \simeq \frac{v^{2}}{\Lambda^{2}}\left(3 y_{t}^{2} c_{+} \lambda_{33}^{u} L_{t}-g_{2}^{2} C_{3} L_{z}+\frac{g_{1}^{2}}{3} C_{1} L_{z}\right) \frac{\lambda_{i j}^{e}}{16 \pi^{2}}$,

with $L_{t}=\log \left(\Lambda / m_{t}\right), L_{z}=\log \left(\Lambda / m_{Z}\right)$, and $\Delta g_{\ell R}=0$. The above expressions provide a good approximation of the exact results, which will be given elsewhere and which have been obtained adding to the RGE contributions from gauge and top Yukawa interactions the explicit one-loop matrix element with the $Z$ four-momentum set on the mass shell. The scale dependence of the RGE contribution cancels with that of the matrix element dominated by a quark loop. Hereafter, we systematically neglect corrections of order $m_{q}^{2} /\left(16 \pi^{2} \Lambda^{2}\right)$ when $q=u, d, c, s, b$.

Quantum effects generate also a purely leptonic effective Lagrangian, as well as corrections to the semileptonic interactions. After running the Wilson coefficients from $\Lambda$ down to the electroweak scale and integrating out the $W$, $Z$, and the heavy quarks $c, b$, and $t$, we get

$$
\begin{aligned}
\mathcal{L}_{\text {eff }}^{\ell}= & -\frac{4 G_{F}}{\sqrt{2}} \lambda_{i j}^{e}\left[\left(\bar{e}_{L i} \gamma_{\mu} e_{L j}\right) \sum_{\psi} \bar{\psi} \gamma^{\mu} \psi\left(2 g_{\psi}^{z} c_{t}^{e}-Q_{\psi} c_{\gamma}^{e}\right)\right. \\
& \left.+c_{t}^{c c}\left(\bar{e}_{L i} \gamma_{\mu} \nu_{L j}\right)\left(\bar{\nu}_{L k} \gamma^{\mu} e_{L k}+\bar{u}_{L k} \gamma^{\mu} V_{k l} d_{L l}\right)+\text { H.c. }\right],
\end{aligned}
$$


where $\psi=\left\{\nu_{L k}, e_{L k, R k}, u_{L, R}, d_{L, R}, s_{L, R}\right\}$, and $g_{\psi}^{z}$ is the fermionic $Z$ coupling defined as $g_{\psi}^{z}=T_{3}(\psi)-Q_{\psi} \sin ^{2} \theta_{W}$. In Eq. (21), we neglected additional neutrino interactions irrelevant in our analysis. Finally, the coefficients $c_{t}^{e, c c}$ and $c_{\gamma}^{e}$ are given by

$$
\begin{aligned}
c_{t}^{e}= & \frac{3 y_{t}^{2}}{32 \pi^{2}} \frac{v^{2}}{\Lambda^{2}}\left(C_{1}-C_{3}\right) \lambda_{33}^{u} \log \frac{\Lambda^{2}}{m_{t}^{2}}, \\
c_{t}^{c c}= & \frac{3 y_{t}^{2}}{16 \pi^{2}} \frac{v^{2}}{\Lambda^{2}} C_{3} \lambda_{33}^{u}\left[\log \frac{\Lambda^{2}}{m_{t}^{2}}+\frac{1}{2}\right], \\
c_{\gamma}^{e}= & \frac{e^{2}}{48 \pi^{2}} \frac{v^{2}}{\Lambda^{2}}\left[\left(3 C_{3}-C_{1}\right) \log \frac{\Lambda^{2}}{\mu^{2}}-\left(C_{1}+C_{3}\right) \lambda_{33}^{d} \log \frac{m_{b}^{2}}{\mu^{2}}\right. \\
& \left.+2\left(C_{1}-C_{3}\right)\left(\lambda_{33}^{u} \log \frac{m_{t}^{2}}{\mu^{2}}+\lambda_{22}^{u} \log \frac{m_{c}^{2}}{\mu^{2}}\right)\right] .
\end{aligned}
$$

The residual scale dependence is removed by evaluating the matrix elements in the low-energy theory, which includes the light quarks $u, d, s$. For simplicity, we have done this within the quark model, by assuming for $u, d$, and $s$ a common constituent mass $\mu \approx 1 \mathrm{GeV}$.

As shown by Eq. (22), $\mathcal{L}_{\text {eff }}^{\ell}$ receives one-loop RGEinduced contributions of order $y_{t}^{2} / 16 \pi^{2}$ and $e^{2} / 16 \pi^{2}$. The former arises from the top-quark Yukawa interactions and affects both the neutral and charged currents. On the contrary, the effects induced by the SM gauge interactions cancel completely in the charged current and only partially in the neutral current, where they are proportional to $e^{2}$ and to the electromagnetic current.

III. Observables.-We proceed by analyzing the phenomenological implications of our low-energy theory. We will revisit first the anomalies in the processes $B \rightarrow K \ell \bar{\ell}$ and $B \rightarrow D^{(*)} \ell \bar{\nu}$ under the constraints imposed by $B \rightarrow K \nu \bar{\nu}$. Then, we will study observables receiving contributions at the loop level, so far overlooked in the literature, which include both LFV and LFU breaking effects in $Z$ and $\tau$ decays.

In our model, $R_{K}^{\mu / e}$ is approximated by the expression

$$
R_{K}^{\mu / e} \approx \frac{\left|C_{9}^{\mu \mu}+C_{9}^{\mathrm{SM}}\right|^{2}}{\left|C_{9}^{e e}+C_{9}^{\mathrm{SM}}\right|^{2}},
$$

where $C_{9}^{\mathrm{SM}} \approx 4.2$. The experimental central value $R_{K}^{\mu / e} \approx$ 0.75 is reproduced for $C_{9}^{\mu \mu} \approx-0.5$ if we assume $C_{9}^{e e}=0$. In particular, we find that

$$
R_{K}^{\mu / e} \approx 1-0.28 \frac{\left(C_{1}+C_{3}\right)}{\Lambda^{2}(\mathrm{TeV})} \frac{\lambda_{23}^{d} \lambda_{22}^{e}}{10^{-3}}
$$

The expression for $R_{D^{(*)}}^{\tau / \ell}$ reads

$$
R_{D^{(*)}}^{\tau / \ell}=\frac{\sum_{j}\left|\left(C_{L}^{c b}\right)_{3 j}\right|^{2}}{\sum_{j}\left|\left(C_{L}^{c b}\right)_{\ell j}\right|^{2}},
$$

where $\ell=e, \mu$. Assuming that $\lambda_{22}^{e} \ll \lambda_{33}^{e} \sim 1$, we find

$$
R_{D^{(*)}}^{\tau / \ell} \approx 1-\frac{0.12 C_{3}}{\Lambda^{2}(\mathrm{TeV})}\left(\lambda_{33}^{d}+\frac{V_{c s}}{V_{c b}} \lambda_{23}^{d}\right) .
$$

The condition $\lambda_{22}^{e} \ll \lambda_{33}^{e}$ is justified by the nonobservation of LFU breaking effects in the $\mu / e$ sector up to the $\lesssim 2 \%$ level [20,21], leading to the upper bound $\lambda_{22}^{e} \lesssim 0.1$ once the anomaly in the $\tau / \ell$ sector is explained. In our estimates, we always set $\lambda_{11}^{d}=0$, as well as $\lambda_{11}^{e}=0$, which implies $\lambda_{22}^{e} \sim\left(\lambda_{23}^{e}\right)^{2}$.

As already noted in Ref. [17], nontrivial constraints arise from the process $B \rightarrow K \nu \bar{\nu}$. Defining $R_{K}^{\nu \nu}$ as

$$
R_{K}^{\nu \nu}=\frac{\mathcal{B}(B \rightarrow K \nu \bar{\nu})}{\mathcal{B}(B \rightarrow K \nu \bar{\nu})_{\mathrm{SM}}}=\frac{\sum_{i j}\left|C_{\nu}^{\mathrm{SM}} \delta^{i j}+C_{\nu}^{i j}\right|^{2}}{3\left|C_{\nu}^{\mathrm{SM}}\right|^{2}},
$$

where $C_{\nu}^{\mathrm{SM}} \approx-6.4$, and exploiting the properties $\operatorname{tr} \lambda^{f}=1$ and $\sum_{i j}\left|\lambda_{i j}^{f}\right|^{2}=1$, we obtain

$R_{K}^{\nu \nu} \approx 1+\frac{0.6 c_{-}}{\Lambda^{2}(\mathrm{TeV})}\left(\frac{\lambda_{23}^{d}}{0.01}\right)+\frac{0.3 c_{-}^{2}}{\Lambda^{4}(\mathrm{TeV})}\left(\frac{\lambda_{23}^{d}}{0.01}\right)^{2}$,

while the experimental bound reads $R_{K}^{\nu \nu}<4.3$ [22]. If LFU effects arise from LFV sources, LFV phenomena are unavoidable [12]. In our setting, it turns out that [23]

$$
\mathcal{B}(B \rightarrow K \tau \mu) \approx 4 \times 10^{-8}\left|C_{9}^{\mu \tau}\right|^{2} \approx 10^{-7}\left|\frac{C_{9}^{\mu \mu}}{0.5} \frac{0.3}{\lambda_{23}^{e}}\right|^{2},
$$

where we have exploited the relation $C_{9}^{\mu \mu} / C_{9}^{\mu \tau} \approx \lambda_{23}^{e}$ and set $\left|C_{9}^{\mu \mu}\right| \approx 0.5$ to accommodate the $R_{K}^{e / \mu}$ anomaly. The above prediction is orders of magnitude below the current bound $\mathcal{B}(B \rightarrow K \tau \mu) \leq 4.8 \times 10^{-5}$ [24].

Modifications of the leptonic $Z$ couplings are constrained by the LEP measurements of the $Z$ decay widths, left-right, and forward-backward asymmetries. The bounds on lepton nonuniversal couplings are [20]

$$
\frac{v_{\tau}}{v_{e}}=0.959(29), \quad \frac{a_{\tau}}{a_{e}}=1.0019(15),
$$

where $v_{\ell}$ and $a_{\ell}$ are the vector and axial-vector couplings, respectively, defined as $v_{\ell}=g_{\ell L}^{\ell \ell}+g_{\ell R}^{\ell \ell}$ and $a_{\ell}=g_{\ell L}^{\ell \ell}-g_{\ell R}^{\ell \ell}$. We get

$$
\frac{v_{\tau}}{v_{e}} \simeq 1-\frac{2 \Delta g_{\ell L}^{33}}{\left(1-4 s_{W}^{2}\right)}, \quad \frac{a_{\tau}}{a_{e}} \simeq 1-2 \Delta g_{\ell L}^{33},
$$

leading to the following numerical estimates

$$
\begin{aligned}
& \frac{v_{\tau}}{v_{e}} \approx 1-0.05 \frac{\left(c_{-}+0.2 C_{3}\right)}{\Lambda^{2}(\mathrm{TeV})}, \\
& \frac{a_{\tau}}{a_{e}} \approx 1-0.004 \frac{\left(c_{-}+0.2 C_{3}\right)}{\Lambda^{2}(\mathrm{TeV})},
\end{aligned}
$$

where we took $\lambda_{33}^{u} \sim \lambda_{33}^{e} \sim 1$ and, hereafter, we set $\Lambda=1 \mathrm{TeV}$ in the argument of the logarithm. Moreover, modifications of the $Z$ couplings to neutrinos affect the extraction of the number of neutrinos $N_{\nu}$ from the invisible $Z$ decay width. We find that 


$$
N_{\nu}=2+\left(\frac{g_{\nu L}^{33}}{g_{\nu L}^{\mathrm{SM}}}\right)^{2} \simeq 3+4 \Delta g_{\nu L}^{33},
$$

leading to the following numerical estimate

$$
N_{\nu} \approx 3+0.008 \frac{\left(c_{+}-0.2 C_{3}\right)}{\Lambda^{2}(\mathrm{TeV})},
$$

to be compared with the experimental result [20]

$$
N_{\nu}=2.9840 \pm 0.0082 \text {. }
$$

Finally, we have checked that $\mathcal{B}\left(Z \rightarrow \mu^{ \pm} \tau^{\mp}\right)$ is always well below the current experimental bound.

LFU breaking effects in $\tau \rightarrow \ell \bar{\nu} \nu$ (with $\ell_{1,2}=e, \mu$ ) are described by the observables

$$
R_{\tau}^{\tau / \ell_{1,2}}=\frac{\mathcal{B}\left(\tau \rightarrow \ell_{2,1} \nu \bar{\nu}\right)_{\exp } / \mathcal{B}\left(\tau \rightarrow \ell_{2,1} \nu \bar{\nu}\right)_{\mathrm{SM}}}{\mathcal{B}(\mu \rightarrow e \nu \bar{\nu})_{\exp } / \mathcal{B}(\mu \rightarrow e \nu \bar{\nu})_{\mathrm{SM}}},
$$

and are experimentally tested at the few \% level [25]

$R_{\tau}^{\tau / \mu}=1.0022 \pm 0.0030, \quad R_{\tau}^{\tau / e}=1.0060 \pm 0.0030$.

We find

$$
R_{\tau}^{\tau / \ell} \simeq 1+2 c_{t}^{c c} \lambda_{33}^{e} \approx 1+\frac{0.008 C_{3}}{\Lambda^{2}(\mathrm{TeV})} .
$$

The effective Lagrangian of Eq. (21) generates LFV processes such as $\tau \rightarrow \mu \ell \ell$ and $\tau \rightarrow \mu P$ with $P=\pi, \eta, \eta^{\prime}, \rho$, etc. The most sensitive channels taking into account their NP sensitivities and experimental resolutions are $\tau \rightarrow \mu \ell \ell$, $\tau \rightarrow \mu \rho$, and $\tau \rightarrow \mu \pi$. For $\tau \rightarrow \mu \ell \ell$, we find

$\frac{\mathcal{B}(\tau \rightarrow \mu \ell \ell)}{\mathcal{B}(\tau \rightarrow \mu \nu \bar{\nu})}=\left|\lambda_{23}^{e}\right|^{2}\left[\left(1+\delta_{\ell \mu}\right)\left(c_{\mathrm{LR}}-c_{t}^{e}\right)^{2}+c_{\mathrm{LR}}^{2}\right]$,

where $c_{\mathrm{LR}}=2 s_{W}^{2} c_{t}^{e}+c_{\gamma}^{e}$. If $c_{-} \sim \mathcal{O}(1)$, we obtain

$$
\mathcal{B}(\tau \rightarrow 3 \mu) \approx 5 \times 10^{-8} \frac{c_{-}^{2}}{\Lambda^{4}(\mathrm{TeV})}\left(\frac{\lambda_{23}^{e}}{0.3}\right)^{2},
$$

while the current bound is $\mathcal{B}(\tau \rightarrow 3 \mu) \leq 1.2 \times 10^{-8}$ [24]. Setting $c_{-}(\Lambda)=0$ leads to $\mathcal{B}(\tau \rightarrow 3 \mu) \approx 4 \times 10^{-9}$ for $\Lambda=1 \mathrm{TeV}, \lambda_{23}^{e}=0.3$, and $C_{1}=C_{3}=1$, yet within the future expected experimental sensitivity. Moreover, it turns out that $1.5 \lesssim \mathcal{B}(\tau \rightarrow 3 \mu) / \mathcal{B}(\tau \rightarrow \mu e e) \lesssim 2$. Finally, employing the general formulas of Ref. [26], we find

$$
\begin{aligned}
\mathcal{B}(\tau \rightarrow \mu \rho) & \approx 2\left|\lambda_{23}^{e}\right|^{2}\left[\left(2 s_{W}^{2}-1\right) c_{t}^{e}+c_{\gamma}^{e}\right]^{2} \mathcal{B}(\tau \rightarrow \nu \rho) \\
& \approx 5 \times 10^{-8} \frac{\left(c_{-}-0.28 C_{3}\right)^{2}}{\Lambda^{4}(\mathrm{TeV})}\left(\frac{\lambda_{23}^{e}}{0.3}\right)^{2},
\end{aligned}
$$

and

$$
\begin{aligned}
\mathcal{B}(\tau \rightarrow \mu \pi) & \approx 2\left|\lambda_{23}^{e}\right|^{2}\left[c_{t}^{e}\right]^{2} \mathcal{B}(\tau \rightarrow \nu \pi) \\
& \approx 8 \times 10^{-8} \frac{c_{-}^{2}}{\Lambda^{4}(\mathrm{TeV})}\left(\frac{\lambda_{23}^{e}}{0.3}\right)^{2},
\end{aligned}
$$

where the current bounds are $\mathcal{B}(\tau \rightarrow \mu \rho) \leq 1.5 \times 10^{-8}$ and $\mathcal{B}(\tau \rightarrow \mu \pi) \leq 2.7 \times 10^{-8}[24]$.

We discuss now the necessary conditions to accommodate the $B$-physics anomalies and their phenomenological implications. Two scenarios are envisaged: (i) $C_{1}=0$ and $C_{3} \neq 0$ and (ii) $C_{1}=C_{3}$. In both cases, the correct pattern of deviation from the SM expectations is reproduced for $C_{3}<0,\left|\lambda_{23}^{d} / V_{c b}\right|<1$, and $\lambda_{23}^{d}<0$; see Eqs. (24) and (26). Moreover, for $\left|C_{3}\right| \sim \mathcal{O}(1)$, the upper bound $\Lambda \lesssim$ $1 \mathrm{TeV}$ and the lower bound $\left|\lambda_{23}^{e}\right| \gtrsim 0.1$ are also predicted. The major differences between the two scenarios concern the impact of the constraints from $Z$-pole and $\tau$ observables. In particular, from Eqs. (30) and (32) we learn that NP effects in $v_{\tau} / v_{e}$ and $a_{\tau} / a_{e}$ are uncomfortably large in scenario (i), while they are under control in (ii). Similarly, $\mathcal{B}(\tau \rightarrow 3 \mu)$ is 1 order of magnitude larger in (i) than in (ii); see Eq. (40) and the following discussion. Most important, we find that $R_{\tau}^{\tau / \ell}$ strongly disfavors an explanation of the $R_{D^{(*)}}^{\tau / \ell}$ anomaly based on left-handed effective operators; see Eqs. (26) and (38). This is confirmed by the upper plot of Fig. 1 (where, to be conservative, we did not impose the
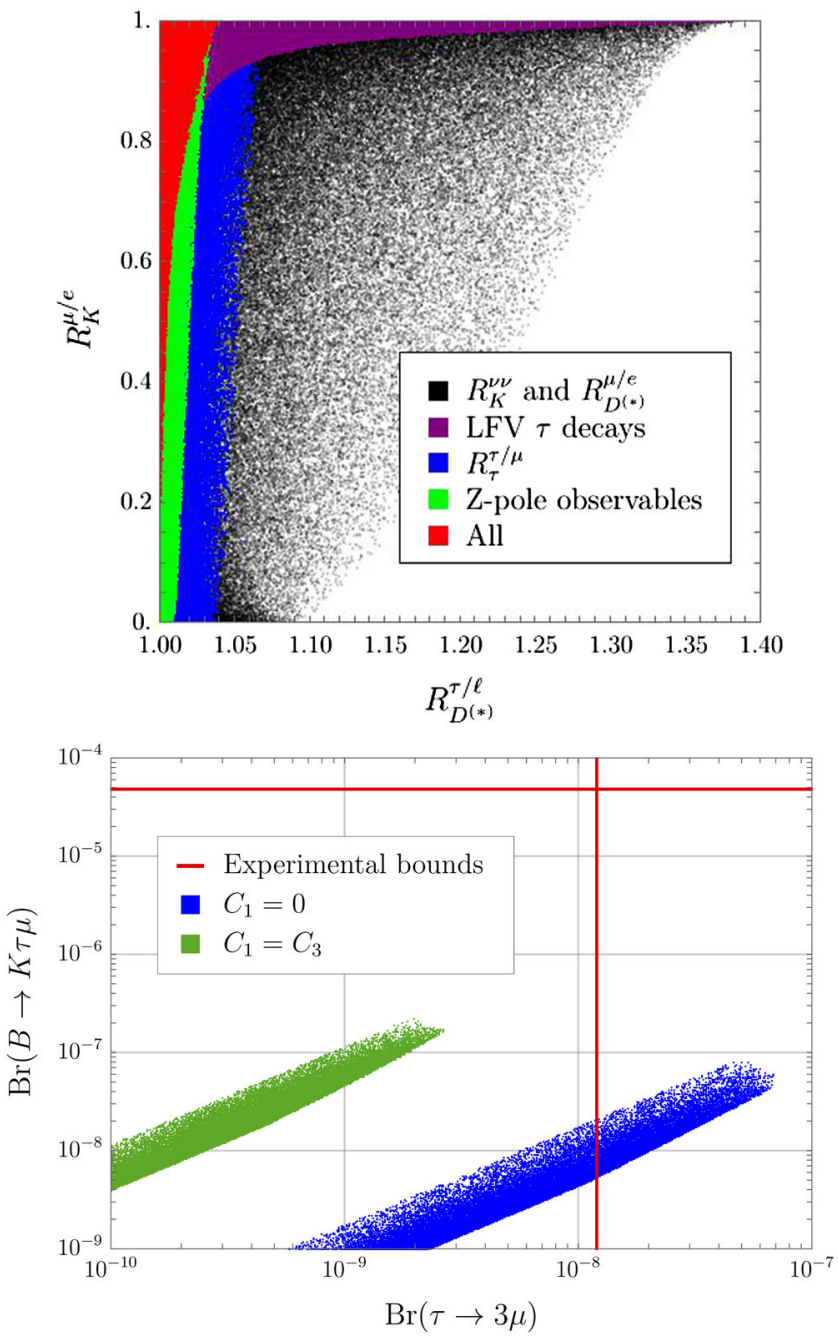

FIG. 1. Upper plot: $R_{K}^{\mu / e}$ vs $R_{D^{(*)}}^{\tau / \ell}$ for $C_{1}=0,\left|C_{3}\right| \leq 3$, $\left|\lambda_{23}^{d}\right| \leq 0.04$, and $\left|\lambda_{23}^{e}\right| \leq 1 / 2$. The allowed regions are colored according to the legend. Lower plot: $\mathcal{B}(B \rightarrow K \tau \mu)$ vs $\mathcal{B}(\tau \rightarrow 3 \mu)$ for $\left|\lambda_{23}^{d}\right|=0.01, C_{1}=C_{3}$ (green points), or $C_{1}=0$ (blue points) imposing all the experimental bounds except $R_{D^{(*)}}^{\tau / \ell}$. 
strong bound from $R_{\tau}^{\tau / e}$ ) showing $R_{K}^{\mu / e}$ vs $R_{D^{(*)}}^{\tau / \ell}$ in scenario (i). The overall picture does not change in scenario (ii) as the $R_{\tau}^{\tau / \mu}$ bound is unchanged. In the lower plot of Fig. 1, we show $\mathcal{B}(B \rightarrow K \tau \mu)$ vs $\mathcal{B}(\tau \rightarrow 3 \mu)$. Considering the current and expected future experimental sensitivities, we conclude that $\tau \rightarrow 3 \mu$ is a more powerful probe than $B \rightarrow K \tau \mu$ of both scenarios, especially scenario (i).

IV. Conclusions. - Recent experimental data hinting at nonstandard LFU breaking effects in semileptonic $B$ decays [1-3,5] stimulated many theoretical investigations of NP scenarios [7-17,27]. In this Letter, we revisited LFU in $B$ decays assuming a class of gauge invariant semileptonic operators at the NP scale $\Lambda \gg v$, as in Refs. [11-17]. We constructed the low-energy effective Lagrangian taking into account the running effects from $\Lambda$ down to $v$ through the one-loop RGEs in the limit of exact electroweak symmetry and QED RGEs from $v$ down to the $1 \mathrm{GeV}$ scale. At the quantum level, we found that the leptonic couplings of the $W$ and $Z$ vector bosons are modified. Moreover, quantum effects generate also a purely leptonic effective Lagrangian, as well as corrections to the semileptonic interactions. The main phenomenological implications of these findings are the generation of large LFU breaking effects in $Z$ and $\tau$ decays, which are correlated with the $B$ anomalies, and $\tau$ LFV processes. Overall, the experimental bounds on $Z$ and $\tau$ decays significantly constrain LFU breaking effects in $B$ decays, challenging an explanation of the current nonstandard data [1-3,5], at least in the framework adopted here. Interestingly, if LFU breaking effects arise from LFV sources, the most sensitive LFV channels are not $B$ decays, as commonly claimed in the literature but, instead, $\tau$ decays such as $\tau \rightarrow \mu \ell \ell$ and $\tau \rightarrow \mu \rho$. Although our results were obtained in the context of an effective Lagrangian dominated by left-handed operators, the present work showed that electroweak radiative effects should be carefully analyzed in any framework addressing the explanation of $B$ anomalies.

This work was supported in part by the MIUR-PRIN Project No. 2010YJ2NYW and by the European Union network FP10 ITN ELUSIVES Grant Nos. (H2020MSCA-ITN-2015-6748896) and INVISIBLES-PLUS (H2020-MSCA-RISA-2015-690575). The research of P. P. is supported by the ERC Advanced Grant No. 267985 (DaMeSyFla), by the research grant TAsP, and by the INFN. The research of A.P. is supported by the Swiss National Science Foundation (SNF) under Contract No. 200021-159720.

[1] J. P. Lees et al. (BABAR Collaboration), Phys. Rev. D 88, 072012 (2013).

[2] M. Huschle et al. (Belle Collaboration), Phys. Rev. D 92, 072014 (2015).

[3] R. Aaij et al. (LHCb Collaboration), Phys. Rev. Lett. 115, 159901 (2015).
[4] S. Fajfer, J. F. Kamenik, and I. Nisandzic, Phys. Rev. D 85, 094025 (2012); H. Na, C. M. Bouchard, G. Peter Lepage, C. Monahan, and J. Shigemitsu (HPQCD Collaboration), Phys. Rev. D 92, 054510 (2015).

[5] R. Aaij et al. (LHCb Collaboration), Phys. Rev. Lett. 113, 151601 (2014).

[6] M. Bordone, G. Isidori, and A. Pattori, Eur. Phys. J. C 76, 440 (2016).

[7] G. Hiller and M. Schmaltz, Phys. Rev. D 90, 054014 (2014).

[8] T. Hurth, F. Mahmoudi, and S. Neshatpour, J. High Energy Phys. 12 (2014) 053.

[9] W. Altmannshofer and D. M. Straub, Eur. Phys. J. C 75, 382 (2015).

[10] S. Descotes-Genon, L. Hofer, J. Matias, and J. Virto, J. High Energy Phys. 06 (2016) 092.

[11] B. Bhattacharya, A. Datta, D. London, and S. Shivashankara, Phys. Lett. B 742, 370 (2015).

[12] S. L. Glashow, D. Guadagnoli, and K. Lane, Phys. Rev. Lett. 114, 091801 (2015).

[13] R. Alonso, B. Grinstein, and J. Martin Camalich, J. High Energy Phys. 10 (2015) 184.

[14] S. Fajfer, J. F. Kamenik, I. Nisandzic, and J. Zupan, Phys. Rev. Lett. 109, 161801 (2012).

[15] R. Alonso, B. Grinstein, and J. Martin Camalich, Phys. Rev. Lett. 113, 241802 (2014).

[16] A. J. Buras, J. Girrbach-Noe, C. Niehof,f, and D. M. Straub, J. High Energy Phys. 02 (2015) 184.

[17] L. Calibbi, A. Crivellin, and T. Ota, Phys. Rev. Lett. 115, 181801 (2015).

[18] E. E. Jenkins, A. V. Manohar, and M. Trott, J. High Energy Phys. 01 (2014) 035; R. Alonso, E. E. Jenkins, A. V. Manohar, and M. Trott, J. High Energy Phys. 04 (2014) 159.

[19] G. Buchalla, A. J. Buras, and M. E. Lautenbacher, Rev. Mod. Phys. 68, 1125 (1996).

[20] K. A. Olive et al. (Particle Data Group Collaboration), Chin. Phys. C 38, 090001 (2014).

[21] A. Greljo, G. Isidori, and D. Marzocca, J. High Energy Phys. 07 (2015) 142.

[22] J. P. Lees et al. (BABAR Collaboration), Phys. Rev. D 87, 112005 (2013).

[23] A. Crivellin, L. Hofer, J. Matias, U. Nierste, S. Pokorski, and J. Rosiek, Phys. Rev. D 92, 054013 (2015); D. Becirevic, O. Sumensari, and R. Z. Funchal, Eur. Phys. J. C 76, 134 (2016).

[24] Y. Amhis et al. (Heavy Flavor Averaging Group Collaboration), arXiv:1412.7515.

[25] A. Pich, Prog. Part. Nucl. Phys. 75, 41 (2014).

[26] A. Brignole and A. Rossi, Nucl. Phys. B701, 3 (2004); A. Celis, V. Cirigliano, and E. Passemar, Phys. Rev. D 89, 095014 (2014).

[27] W. Altmannshofer, S. Gori, M. Pospelov, and I. Yavin, Phys. Rev. D 89, 095033 (2014); B. Gripaios, M. Nardecchia, and S. A. Renner, J. High Energy Phys. 05 (2015) 006; C. Niehoff, P. Stangl, and D. M. Straub, Phys. Lett. B 747, 182 (2015); D. Becirevic, S. Fajfer, and N. Kosnik, Phys. Rev. D 92, 014016 (2015); M. Freytsis, Z. Ligeti, and J. T. Ruderman, Phys. Rev. D 92, 054018 (2015); M. Bauer and M. Neubert, Phys. Rev. Lett. 116, 141802 (2016); S. Fajfer and N. Konik, Phys. Lett. B 755, 270 (2016); A. Falkowski, M. Nardecchia, and R. Ziegler, J. High Energy Phys. 11 (2015) 173; R. Barbieri, G. Isidori, A. Pattori, and F. Senia, Eur. Phys. J. C 76, 67 (2016). 